\title{
THE SPECTRAL THEOREM IN BANACH ALGEBRAS
}

\section{by STEPHEN PLAFKER}

(Received 9 March, 1970; revised 30 June, 1970)

Introduction. The concept of a hermitian element of a Banach algebra was first introduced by Vidav [21] who proved that, if a Banach algebra $\mathscr{A}$ has " enough" hermitian elements, then $\mathscr{A}$ can be renormed and given an involution to make it a stellar algebra. (Following Bourbaki [5] we shall use the expression "stellar algebra " in place of the term " $C$ *-algebra".) This theorem was improved by Berkson [2], Glickfeld [10] and Palmer [17]. The improvements consist of removing hypotheses from Vidav's original theorem and in showing that Vidav's new norm is in fact the original norm of the algebra. Lumer [13] gave a spatial definition of a hermitian operator on a Banach space $E$ and proved it to be equivalent to Vidav's definition when one considers the Banach algebra $\mathscr{L}(E)$ of continuous linear mappings of $E$ into $E$.

In this paper the theory outlined above will be applied to define a normal element of a Banach algebra and to prove a spectral theorem for such elements. This theorem will then be exploited to prove analogues of well-known theorems for operators in Hilbert spaces.

We shall use the following standard notations. The symbol $\mathbf{N}$ will denote the set $\{0,1,2, \ldots\}, \mathbf{R}$ the set of real numbers, $\mathbf{C}$ the set of complex numbers, $\mathbf{T}^{\mathbf{1}}$ the unit circle in $\mathbf{C}$, and $z$ the identity function of $\mathbf{R}^{2}$ onto $\mathbf{R}^{2}$.

The Banach algebras considered here will be assumed to be complex and to have identity element 1 such that $\|1\|=1$. For an element $x$ of a Banach algebra $\mathscr{A}$, the spectrum of $x$, denoted by $\operatorname{sp}(x)$, is the set of complex numbers $\lambda$ such that $\lambda-x(=\lambda 1-x)$ is not invertible in $\mathscr{A}$. The spectral radius of $x$ is the number

$$
\rho(x)=\sup \{|\lambda|: \lambda \in \operatorname{sp}(x)\} .
$$

Note that $\rho(x) \leqq\|x\|$.

Let $\mathscr{A}$ be a Banach algebra, and let $x \in \mathscr{A}$. Since the mapping $t \rightarrow\|1+t x\|$ is a convex function of $\mathbf{R}$ into $\mathbf{R}$, one can define

$$
\varphi(x)=\lim _{t \rightarrow 0+} \frac{\|1+t x\|-1}{t}
$$

An element $x \in \mathscr{A}$ is hermitian if $\varphi(i x)=\varphi(-i x)=0 ; x$ is positive if $x$ is hermitian and has positive spectrum. Since, for $y \in \mathscr{A}, \varphi(y)+\varphi(-y) \geqq 0, x$ is hermitian if both $\varphi(i x)$ and $\varphi(-i x)$ are negative and positive if, in addition, $\varphi(-x) \leqq 0$. If $\mathscr{A}$ is a stellar algebra, $x$ is hermitian (in the sense above) if and only if $x$ is self-adjoint $\left(x=x^{*}\right)$. The function $\Phi$ of $\mathscr{A}$ into $\mathbf{R}$ defined by the equation

$$
\Phi(x)=\sup \{\varphi(\lambda x): \lambda \in \mathbf{C},|\lambda| \leqq 1\}
$$

is a norm on $\mathscr{A}$ equivalent to the original norm. The above facts are proved in [4].

We shall list here the basic facts that we shall use throughout the paper.

Proposition A. The element $x \in \mathscr{A}$ is hermitian if and only if $\left\|e^{i t x}\right\|=1$ for every $t \in \mathbf{R}$.

D 
Proposimon B [21, Hilfsatz 2 (e)]. If $x \in \mathscr{A}$ is hermitian, $\mathrm{sp}(x) \subset \mathbf{R}$.

Proposition C [2, Lemma 3.1]. Let $H$ be the set of hermitian elements of $\mathscr{A}$. Then $H+i H$ is closed in $\mathscr{A}$.

Proof. First note that, if $a, b \in H$, then $\varphi(a+i b) \leqq \varphi(a)+\varphi(i b)=\varphi(a)=\varphi(a+i b-i b) \leqq$ $\varphi(a+i b)+\varphi(-i b)=\varphi(a+i b)$; hence $\varphi(a+i b)=\varphi(a)$. Consequently $\Phi(a) \leqq \Phi(a+i b)$.

Now let $\left(x_{n}\right)$ be a sequence in $H+i H$ converging to $x \in \mathscr{A}$. Write $x_{n}=a_{n}+i b_{n}, a_{n}, b_{n} \in H$. By the above discussion $\left(a_{n}\right)$ is a Cauchy sequence (for $\Phi$ and therefore for the original norm). Thus the sequence $\left(a_{n}\right)$ [resp. $\left(b_{n}\right)$ ] converges to $a \in \mathscr{A}$ [resp. $b \in \mathscr{A}$ ]. But $H$ is closed [21, Hilfsatz 2(d)]; hence $x=(a+i b) \in H+i H$.

Proposition D [21, II]. If $\mathscr{A}$ and $\mathscr{B}$ are Banach algebras, and $u$ is a norm-decreasing linear mapping of $\mathscr{B}$ into $\mathscr{A}$ mapping 1 onto 1 , then $u$ carries hermitian [resp. positive] elements of $\mathscr{B}$ onto hermitian [resp. positive] elements of $\mathscr{A}$.

Proof. For any $y \in \mathscr{B}, \varphi(u(y)) \leqq \varphi(y)$. Hence, for $x \in \mathscr{B}$ hermitian, $\varphi(i u(x)) \leqq \varphi(i x)=0$ and $\varphi(-i u(x)) \leqq \varphi(-i x)=0$; furthermore, for $x$ positive, $\varphi(-u(x)) \leqq \varphi(-x) \leqq 0$.

Proposition E [21, Hilfsatz 2(c)]. If $a+i b=a^{\prime}+i b^{\prime}$ where $a, b, a^{\prime}$, and $b^{\prime}$ are hermitian, then $a=a^{\prime}$ and $b=b^{\prime}$.

Proposition F [17, Theorem]. If $\mathscr{A}$ is (algebraically) spanned by its hermitian elements (i.e. $\mathscr{A}=H+i H)$, the mapping $x \rightarrow x^{*}$ is an involution on $\mathscr{A}$ under which $\mathscr{A}$ becomes a stellar algebra. (If $x=a+i b\left(a, b\right.$ hermitian) then $x^{*}=a-i b$.)

Proposition G [21]. If $x \in \mathscr{A}$ is hermitian and quasi-nilpotent $(\operatorname{sp}(x)=\{0\})$, then $x=0$.

1. The spectral theorem for normal elements of a Banach algebra. In this section we shall introduce the concept of a normal element of a Banach algebra and prove a spectral theorem for such elements. This theorem depends on the theory of $C^{\prime}\left(\mathbf{R}^{2}\right)$-scalar elements (a concept due to Foias (see [6]) and Maeda [16]; see [20] for a complete exposition and for further references).

We shall denote by $C^{l}\left(\mathbf{R}^{2}\right)$ the Banach algebra of continuous, complex-valued functions defined on $\mathbf{R}^{2}$ having limits at $\infty$ and by $\mathscr{K}\left(\mathbf{R}^{2}\right)$ the set of continuous functions with compact support. Note that $C^{\prime}\left(\mathbf{R}^{2}\right)$ is a stellar algebra, and that it can be identified with the direct sum of $C_{0}\left(\mathbf{R}^{2}\right)$ and $\mathbf{C}$ where $C_{0}\left(\mathbf{R}^{2}\right)$ is the stellar algebra of continuous functions on $\mathbf{R}^{2}$ vanishing at $\infty$.

Definition. An element $x$ of a Banach algebra $\mathscr{A}$ is $C^{\prime}\left(\mathbf{R}^{2}\right)$-scalar if there exists a continuous representation $u$ of $C^{\prime}\left(\mathbf{R}^{2}\right)$ into $\mathscr{A}$ mapping 1 onto 1 and such that, for every $f \in \mathscr{K}\left(\mathbf{R}^{2}\right)$ taking the value 1 on $\operatorname{sp}(x), u(f)=1$ and $u(z f)=x$.

REMARK. It follows from [6, Corollary 1.6, p. 98] that, for any $C^{\prime}\left(\mathbf{R}^{2}\right)$-scalar element $x \in \mathscr{A}$, there is only one representation $u$ as described in the definition above. It is called the $C^{\prime}\left(\mathbf{R}^{2}\right)$-scalar representation for $x$. Furthermore, for any $f, g \in C^{l}\left(\mathbf{R}^{2}\right)$ which agree on $\mathrm{sp}(x)$, $u(f)=u(g)[6$, Theorem 1.6, p. 60]. 
If $\mathscr{A}$ and $\mathscr{B}$ are Banach algebras, a linear mapping of $\mathscr{B}$ into $\mathscr{A}$ is involutive if it maps hermitian elements of $\mathscr{B}$ into hermitian elements of $\mathscr{A}$.

PROPOSITION 1.1. Let $\mathscr{A}$ be a Banach algebra, $\mathscr{B}$ a stellar algebra, and $u$ a continuous representation of $\mathscr{B}$ into $\mathscr{A}$ mapping 1 onto 1 . Then $u$ is involutive if and only if $u$ has norm 1 .

Proof. If $u$ has norm 1 , use Proposition $\mathrm{D}$ to conclude that $u$ is involutive. To prove the converse consider the closure, $\mathscr{A}^{\prime}$, of the image of $u$. Since $\mathscr{B}$ is (algebraically) spanned by its hermitian elements, and since $u$ is involutive, $\mathscr{A}^{\prime}$ is spanned by its hermitian elements (use Proposition C). Thus, by Proposition F, $\mathscr{A}^{\prime}$ is a stellar algebra. A standard result from the theory of stellar algebras [5, Proposition 1, p. 66] now applies.

Definition. Let $\mathscr{A}$ be a Banach algebra. An element $x \in \mathscr{A}$ is normal if there exist commuting elements $a, b \in \mathscr{A}$ such that

(1) $a^{m} b^{n}$ is hermitian for every $m, n \in \mathbf{N}$;

(2) $x=a+i b$.

We shall call $a$ the real part of $x$ and $b$ the imaginary part of $x$. (Note that, by Proposition E, $a$ and $b$ are unique.)

If $E$ is a Banach space, an operator $T$ on $E$ is normal if $T$ is normal as an element of the Banach algebra $\mathscr{L}(E)$.

Lemma 1.1. Let $X$ be a compact Hausdorff space, $\mathscr{A}$ a Banach algebra, and $x \in \mathscr{A}$. Suppose that there exists a continuous representation $v$ of $C(X)$ into $\mathscr{A}$ which has 1 and $x$ in its image (in particular $v(1)=1$ ). Then there exists a $C^{1}\left(\mathbf{R}^{2}\right)$-scalar representation $u$ for $x$ such that $\|u\| \leqq\|v\|$.

Proof. Define $u(f)=v(f \circ h)$, where $h \in C(X)$ is such that $x=v(h)$.

THEOREM 1.1 (The spectral theorem in Banach algebras). An element $x \in \mathscr{A}$ is normal if and only if $x$ is $C^{l}\left(\mathbf{R}^{2}\right)$-scalar and the $C^{l}\left(\mathbf{R}^{2}\right)$-scalar representation for $x$ has norm 1 .

Proof. First suppose that $x$ is normal. Let $a$ be the real part of $x, b$ the imaginary part of $x$, and let $\mathscr{B}$ be the smallest closed subalgebra of $\mathscr{A}$ containing the set $\{a, b, 1\}$. By Proposition F and the Gelfand Isomorphism Theorem [5, Théorème 1, p. 67] there exists an isometric isomorphism $v$ of $C(\operatorname{sp}(x))$ onto $\mathscr{B}$. One now uses Lemma 1.1 to obtain the desired conclusion.

To prove the converse suppose that $u$ is the $C^{\prime}\left(\mathbf{R}^{2}\right)$-scalar representation of $x$ (so that $\|u\|=1)$. Let $r$ and $s$ be elements of $C^{l}\left(\mathbf{R}^{2}\right)$ such that

$$
r(z)=\mathscr{R}(z) \text { and } s(z)=\mathscr{F}(z)
$$

for every $z \in \operatorname{sp}(x)$, and let

$$
a=u(r) \text { and } b=u(s) .
$$

Clearly $x=a+i b$. For $m, n \in \mathbf{N}, a^{m} b^{n}=u\left(r^{m} s^{n}\right)$ is hermitian by Proposition D. 
COROLlaRY 1. An element $x \in \mathscr{A}$ is normal if and only if $x$ is $C^{l}\left(\mathbf{R}^{2}\right)$-scalar and the $C^{\prime}\left(\mathbf{R}^{2}\right)$-scalar representation for $x$ is involutive.

COROllaRy 2. Let $E$ be a Banach space. A necessary and sufficient condition for an operator $T \in \mathscr{L}(E)$ to be $C^{\prime}\left(\mathbf{R}^{2}\right)$-scalar is that there exist a norm on $E$, equivalent to the original norm, under which $T$ is normal.

Proof. The sufficiency follows easily from previous results. To prove necessity suppose that $T$ is $C^{l}\left(\mathbf{R}^{2}\right)$-scalar and let $U$ be the $C^{l}\left(\mathbf{R}^{2}\right)$-scalar representation for $T$. By Theorem 1.1 we need only exhibit an equivalent norm on $E$ such that, when $E$ is endowed with the new norm, $\|U\|=1$. Such a norm is given by

$$
x \rightarrow \sup \{\|U(f) x\|:\|f\| \leqq 1\} .
$$

REmark. Corollary 2 above is valid in the context of Hilbert spaces [15, 22]. However, the proof given above can not be used in this case since the new norm need not be a Hilbert space norm.

COROLlaRY 3. If $x \in \mathscr{A}$ is normal, then $\|x\|=\rho(x)$.

Proof. Let $u$ be the $C^{\prime}\left(\mathbf{R}^{2}\right)$-scalar representation for $x$, and choose $f \in C^{\prime}\left(\mathbf{R}^{2}\right)$ of norm $\rho(x)$ and such that $f(z)=z$ for $z \in \operatorname{sp}(x)$. Then $\|x\|=\|u(f)\| \leqq\|f\|=\rho(x)$.

COROLlaRY 4 [2, Theorem 2.1]. If $p_{1}, \ldots, p_{n}$ are non-zero disjoint projections (hermitian idempotents) in a Banach algebra $\mathscr{A}$ and $\lambda_{1}, \ldots, \lambda_{n}$ are complex numbers, then

$$
\left\|\sum_{i=1}^{n} \lambda_{i} p_{i}\right\|=\sup \left\{\left|\lambda_{i}\right|: 1 \leqq i \leqq n\right\}
$$

In particular, if $p$ is a non-trivial projection, $\|p\|=\|1-p\|=1$.

Proof. For $f \in C^{\prime}\left(\mathbf{R}^{2}\right)$ define $u(f) \in \mathscr{A}$ by

$$
u(f)=\sum_{i=1}^{n} f\left(\lambda_{i}\right) p_{i}+f(0)\left(1-\sum_{i=1}^{n} p_{i}\right)
$$

Then $u$ is an involutive $C^{l}\left(\mathbf{R}^{2}\right)$-scalar representation for $x=\sum_{i=1}^{n} \lambda_{i} p_{i}$. By Proposition 1.1 and Theorem 1.1, $x$ is normal. By Corollary 3,

$$
\|x\|=\rho(x)=\sup \left\{\left|\lambda_{i}\right|: 1 \leqq i \leqq n\right\} .
$$

REMARK. A necessary and sufficient condition for an idempotent $p \in \mathscr{A}$ to be a projection is that $\|p+\lambda(1-p)\|=1$ for every $\lambda \in \mathbf{T}^{1}$. To prove this fact simply use the equality

$$
\left\|e^{i t p}\right\|=\left\|e^{i t}\left(p+e^{-i t}(1-p)\right)\right\|=\left\|p+e^{-i t}(1-p)\right\|
$$

together with Proposition A. This equivalence was first noted by Palmer [18]. 
EXAmple. If $E=\mathbf{C}^{2}$ with the norm $(x, y) \rightarrow|x|+|y|$ and $P$ is the idempotent in $\mathscr{L}(E)$ defined by

$$
P(x, y)=\frac{1}{2}(x+y, x+y) \text {, }
$$

$\|P\|=\|I-P\|=1$. However

$$
\|(P+i(I-P))(1,0)\|=\sqrt{ } 2 .
$$

Hence $P$ is not hermitian.

E. Berkson has shown [3] that the above example is valid when $\mathbf{C}^{2}$ is endowed with the " $p$-norm" for any $p$ such that $1 \leqq p \leqq \infty, p \neq 2$.

For the next two results we shall need two definitions. An element $x$ of a Banach algebra $\mathscr{A}$ is power hermitian if $x^{n}$ is hermitian for every $n \in \mathbf{N}$. The element $x$ is unitary if $x$ is normal and invertible and if both $x$ and $x^{-1}$ have norm 1 . If $\mathscr{A}$ is a stellar algebra, then every hermitian element is power hermitian. However, there is an example of a hermitian operator on a Banach space which is not power hermitian [14].

Proposition 1.2 [4]. If $a$ and $b$ are commuting hermitian elements of a Banach algebra $\mathscr{A}$, and if $x=a+i b$ has real spectrum, then $x$ is hermitian $(i . e . b=0)$. Consequently, the element $x \in \mathscr{A}$ is power hermitian if and only if $x$ is normal and has real spectrum.

Proof. The hypotheses of the proposition imply that $\rho\left(e^{-i a}\right) \rho\left(e^{b}\right)=\rho\left(e^{-i x}\right)=1$; hence, by [5, Cor. to Prop. 5, p. 26] and the fact that $\rho\left(e^{i a}\right)=\rho\left(e^{-i a}\right)=1, \rho\left(e^{b}\right)=1$. Similarly, $\rho\left(e^{-b}\right)=1$. By the Spectral Mapping Theorem, $b$ is quasi-nilpotent and therefore 0 by Proposition G.

Proposition 1.3 (see [19]). A normal element $x$ of a Banach algebra $\mathscr{A}$ is unitary if and only if $\operatorname{sp}(x) \subset \mathrm{T}^{1}$.

Proof. The fact that a unitary element has a spectrum contained in $\mathbf{T}^{1}$ follows from the Spectral Mapping Theorem. To prove the converse, let $u$ be the $C^{l}\left(\mathbf{R}^{2}\right)$-scalar representation for $x$, and let $f \in C^{1}\left(\mathbf{R}^{2}\right)$ be the identity on $\mathbf{T}^{1}$ and have norm 1. From the fact that $f f=1$ on $\mathrm{sp}(x), x^{-1}=u(f)$; the result follows. of [19].

The next proposition, which we shall state here without proof, is analogous to Theorem 1

Proposition 1.4. If $x$ is a normal, invertible element of a Banach algebra $\mathscr{A}$, then there exist a positive, power hermitian element $y \in \mathscr{A}$ and a unitary element $z \in \mathscr{A}$ such that $x=y z$. Furthermore, if $a$ is the real part of $z$ and $b$ is the imaginary part of $z$, then $y, a$, and $b$ commute and $y^{k} a^{m} b^{n}$ is hermitian for every $k, m, n \in \mathbf{N}$.

If $x \in \mathscr{A}$ is $B^{\infty}\left(\mathbf{R}^{2}\right)$-scalar, the assumption of invertibility can be omitted from the hypotheses of Proposition 1.4. (For the definition of $B^{\infty}\left(\mathbf{R}^{2}\right)$ see $\S 2$.)

2. Scalar operators. In this section we shall examine the preceding results in the context of the scalar operators of Dunford $[7,8]$. We shall begin by reviewing some notation and known theorems, most of which are taken from [11].

We shall let $B^{\infty}\left(\mathbf{R}^{2}\right)$ denote the set of bounded Borel-measurable functions from $\mathbf{R}^{2}$ into C. With the usual addition, multiplication, involution and norm, $B^{\infty}\left(\mathbf{R}^{2}\right)$ is a stellar algebra, 
and $C^{l}\left(\mathbf{R}^{2}\right)$ is a stellar subalgebra of $B^{\infty}\left(\mathbf{R}^{2}\right)$. For any subset $A$ of $\mathbf{R}^{2}, \varphi_{A}$ will denote the characteristic function of $A ; A$ is a Borel set if and only if $\varphi_{A} \in B^{\infty}\left(\mathbf{R}^{2}\right)$. If $A$ is a Borel subset of $\mathbf{R}^{2}$ and if $U$ is a function whose domain is $B^{\infty}\left(\mathbf{R}^{2}\right)$, we shall use the symbol $U_{A}$ in place of $U\left(\varphi_{A}\right)$. Note that, if $U$ is a representation of $B^{\infty}\left(\mathbf{R}^{2}\right)$ into a ring $\mathscr{A}, U_{A}$ is idempotent.

Let $E$ be a Banach space. A continuous representation $U$ of $B^{\infty}\left(\mathbf{R}^{2}\right)$ into $\mathscr{L}(E)$ is standard if, for any bounded sequence $\left(f_{n}\right)$ in $B^{\infty}\left(\mathbf{R}^{2}\right)$ converging pointwise to 0 , the sequence $\left(U\left(f_{n}\right)\right)$ converges strongly to 0 in $\mathscr{L}(E)$. An operator $T \in \mathscr{L}(E)$ is scalar if there exists a standard representation $U$ of $B^{\infty}\left(\mathbf{R}^{2}\right)$ into $\mathscr{L}(E)$ mapping 1 onto $I$ and such that, for every bounded Borel subset $A$ of $\mathbf{R}^{2}, U\left(z \varphi_{A}\right)=U_{A} T$. (These operators were called scalar-type by Dunford [8].)

If $T \in \mathscr{L}(E)$ is scalar, there is only one standard representation that has the properties listed above. It is called the spectral representation for $T$. Furthermore, $U(z f)=U(f) T$ for every $f \in B^{\infty}\left(\mathbf{R}^{2}\right)$ with compact support.

The following theorem, which is proved in [12], summarizes the relationship between scalar and $C^{l}\left(\mathbf{R}^{2}\right)$-scalar operators in weakly complete Banach spaces. A proof, based on the theory of spectral measures as developed in [11], can be given.

THEOREM 2.1. On a weakly complete Banach space $E$, an operator $T \in \mathscr{L}(E)$ is scalar if and only if it is $C^{l}\left(\mathbf{R}^{2}\right)$-scalar. Furthermore, if $U$ is the spectral representation for $T$, then the restriction of $U$ to $C^{l}\left(\mathbf{R}^{2}\right)$ is the $C^{l}\left(\mathbf{R}^{2}\right)$-scalar representation for $T$.

COROLLARY 1. If $T \in \mathscr{L}(E)$ is scalar and if $U$ is the spectral representation for $T$, then the following assertions are equivalent:

(1) $T$ is normal.

(2) $U$ has norm 1.

(3) $U(f)$ is hermitian for every real $f \in B^{\infty}\left(\mathbf{R}^{2}\right)$.

(4) $U_{A}$ is hermitian for every Borel subset of $\mathbf{R}^{2}$.

COROLlary 2 [1, Theorem 4.2; 9]. Let $E$ be a weakly complete Banach space. An operator $T \in \mathscr{L}(E)$ is scalar if and only if there is a norm on $E$, equivalent to the original norm, under which $T$ is normal.

The following corollary follows easily from Theorem 2.1 and Corollary 1.

COROLLARY 3 (The spectral theorem in Hilbert spaces). If $T$ is a normal operator on a Hilbert space $H$, then $T$ is scalar. Furthermore, if $U$ is the spectral representation for $T$, then $U_{A}$ is hermitian for every Borel subset $A$ of $\mathbf{R}^{2}$.

Example. Let $E=C(K)$ where $K$ is an infinite compact subset of $\mathbf{R}^{2}$. For every $g \in C\left(\mathbf{R}^{2}\right)$ define $U(g) \in \mathscr{L}(E)$ by $U(g) x=g x(x \in E)$, and let $T=U(z)$. Then the restriction of $U$ to $C^{l}\left(\mathbf{R}^{2}\right)$ is a $C^{l}\left(\mathbf{R}^{2}\right)$-scalar representation for $T$. (As a matter of fact $U$ has a norm 1 and therefore $T$ is normal.)

On the other hand, let $\left(t_{n}\right)$ be a discrete convergent sequence in $K$. Let $\left(Q_{n}\right)$ be a sequence of open subsets of $\mathbf{R}^{2}$ such that the sequence of closures is disjoint and such that $t_{n} \in Q_{n}$ for every $n \in \mathbf{N}$. For each $n \in \mathbf{N}$, let $f_{n}$ be a unit vector in $E$ with support contained in $Q_{n}$. Then $\left(f_{n}\right)$ is a bounded sequence in $B^{\infty}\left(\mathbf{R}^{2}\right)$ converging pointwise to 0 , but the sequence $\left(U\left(f_{n}\right) 1\right)$ does not converge in $E$. Consequently, $T$ is not scalar. 


\section{REFERENCES}

1. E. Berkson, A characterization of scalar type operators on reflexive Banach spaces, Pacific J. Math. 13 (1963), 365-373.

2. E. Berkson, Some characterizations of $C^{*}$-algebras, Illinois J. Math. 10 (1966), 1-8.

3. E. Berkson, Hermitian projections and orthogonality in Banach spaces, Proc. London Math. Soc. (3) 24 (1972), 101-118.

4. H. Bohnenblust and S. Karlin, Geometrical properties of the unit sphere of Banach algebras, Ann. of Math. 62 (1955), 217-229.

5. N. Bourbaki, Theories spectrales, Chapitres 1 et 2 (Paris, 1967).

6. I. Colojoara and C. Foias, Theory of generalized spectral operators (New York, 1968).

7. N. Dunford, Spectral operators, Pacific J. Math. 4 (1954), 321-354. 217-274.

8. N. Dunford, A survey of the theory of spectral operators, Bull. Amer. Math. Soc. 64 (1958),

9. S. Foguel, The relations between a spectral operator and its scalar part, Pacific J. Math. 8 (1958), 51-65.

10. B. Glickfeld, A metric characterization of $C(X)$ and its generalization to $C^{*}$-algebras, Illinois J. Math. 10 (1966), 547-556.

11. C. Ionescu Tulcea, Notes on spectral theory, Technical report, 1964.

12. S. Kantorovitz, Classification of operators by means of their operational calculus, Trans. Amer. Math. Soc. 115 (1965), 194-224.

13. G. Lumer, Semi-inner-product spaces, Trans. Amer. Math. Soc. 100 (1961), 29-43.

14. G. Lumer, Spectral operators, hermitian operators and bounded groups, Acta. Sci. Math. XXV (1964), 75-85.

15. G. Mackey, Commutative Banach algebras (Rio de Janeiro, 1959).

16. F.-Y. Maeda, Generalized spectral operators on locally convex spaces, Pacific J. Math. 13 (1963), 177-192.

17. T. Palmer, Characterization of C*-algebras, Bull. Amer. Math. Soc. 74 (1968), 538-540.

18. T. Palmer, Unbounded normal operators on Banach spaces, Trans. Amer. Math. Soc. 133 (1968), 385-414.

19. T. Panchapagesan, Unitary operators in Banach spaces, Pacific J. Math. 22 (1967), 465-475.

20. S. Plafker, Spectral representations for a general class of operators on a locally convex space, Illinois J. Math. 13 (1969), 573-582.

21. I. Vidav, Eine metrische Kennzeichnung der selbstadjungierten Operatoren, Math. Zeit. 66 (1956), 121-128.

22. J. Wermer, Commuting spectral measures on Hilbert space, Pacific J. Math. 4 (1954), 355-361.

TULANE UNIVERSITY

New OrLeans, Louisiana 70118 